\title{
Stern-en segidaren propietate berri bat eta segidaren n. gaia azkar kalkulatzeko algoritmo bat
}

\author{
A new property of Stern's sequence and a fast algorithm for \\ calculating the nth term of the sequence
}

\author{
Yosu Yurramendi Mendizabal* \\ Konputazio Zientziak eta Adimen Artifiziala saila, \\ Informatika Fakultatea, Euskal Herriko Unibertsitatea (UPV/EHU)
}

LABURPENA: Stern-en segida zenbaki arruntez osatuta dago, eta propietate asko ditu. Segidaren propietate berri bat azaltzen da lan honetan, hain zuzen segidaren $\mathrm{n}$. gaia zein den azkar kalkulatzeko balio duena. Azkartasun hori n-k sistema bitarrean duen adierazpidean oinarritzen da. Propietatea nondik nora sortu den azaltzen da, baita haren egiazkotasunaren froga matematikoa ere.

HITZ GAKOAK: Stern-en segida, errekurtsibitatea, matematika esperimentala.

ABSTRACT: Stern's sequence consists of natural numbers and has many properties. A new property of the sequence is given in this work, by which the value of the nth term is quickly calculated. This quickness is based on the representation of $n$ in the binary system. It is explained how the property has arisen, as well as the mathematical proof of its truth.

KEYWORDS: Stern sequence, recursion, experimental mathematics.

\footnotetext{
* Harremanetan jartzeko / Corresponding author: Yosu Yurramendi Mendizabal. Konputazio Zientziak eta Adimen Artifiziala saila, Informatika Fakultatea, Euskal Herriko Unibertsitatea, Lardizabal pasealekua, 1, 20018 Donostia. - yosu.yurramendi@ ehu.eus - http://orcid.org/0000-0002-4399-600X.

Nola aipatu / How to cite: Yurramendi Mendizabal, Yosu (2019). «Stern-en segidaren propietate berri bat eta segidaren n. gaia azkar kalkulatzeko algoritmo bat»; Ekaia, 35, 2019, 325-339. (https://doi.org/10.1387/ekaia.19513).

Jasoa: 21 martxoa, 2018; Onartua: 7 abendua, 2018.

ISSN 0214-9001 - elSSN 2444-3255 / (c) 2019 UPV/EHU
}

cc)(1)(2) Obra hau Creative Commons Atribución 4.0 Internacional-en lizentziapean dago 


\section{SARRERA}

Moritz Abraham Stern (1807-1894) matematikari alemaniar bat izan zen, eta haren doktorego tesiaren zuzendaria Carl Friedrich Gauss izan zen [1]. Erreferentzia horretan agertzen da, era berean, Bernhard Riemann-en biografiaren egileak aipatzen duena, hau da, Stern-en kalkulurako ikasbideak gerora zein garrantzia izan zuen harengan. Stern, beraz, lehenengo lerroko matematikaria izan zen.

Stern-en lan ezagunen artean Stern-en segida izena duena dago [2]. Berak azaltzen du lan horren hasiera-hasieran Gauss-en inguruko Frank Gotthold Max Eisenstein gazteak 1850ean jarritako problema bati jarraitzen ari zela.

Stern-ek, aipatutako artikuluan, segida definitzen du, lerroz lerro: lehenengo lerroan bi ' 1 'eko ipintzen ditu, bigarrenean bi ' 1 'eko horiek eta bi ' 1 'koen batura den '2'koa haien tartean, hirugarrenean aurreko lerroko zenbaki guztiak eta hurrenez hurren dauden bi zenbakien artean haien batura, eta abar (ikus 1. taula). Bi zenbakiren baturari mediant edo bitartekari-kokapena esaten zaio.

1. taula. Stern-en segidaren sortze-prozedura.

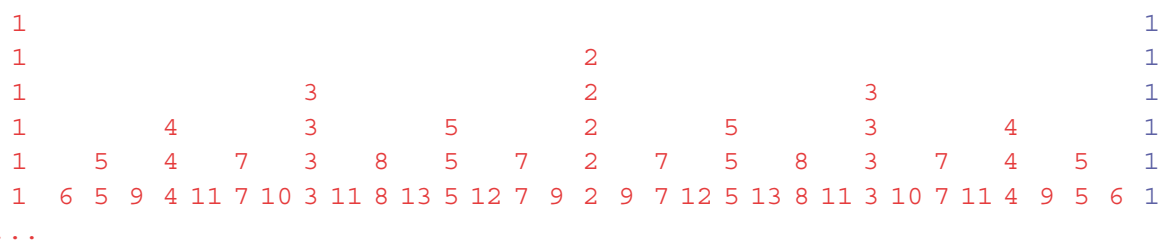

Definizio-prozedura honek badu, berez, Pascal-en triangelua izeneko irudikapen matematikoarekin zerikusirik: lerro berean hurrenez hurren dauden bi zenbakiren batura hurrengo lerroan kokatzen da, haien erdian.

' 1 '-ekoez osatutako eskuineko zutabea ezabatzen bada, eta eraiketaprozedura formalizatzen bada, definizio-formula honetara irits daiteke:

$$
\begin{aligned}
& \mathrm{a}(1)=1, \text { eta } \forall \mathrm{n}>0: \\
& \mathrm{a}(2 \mathrm{n} \quad)=\mathrm{a}(\mathrm{n}) \\
& \mathrm{a}(2 \mathrm{n}+1)=\mathrm{a}(\mathrm{n})+\mathrm{a}(\mathrm{n}+1)
\end{aligned}
$$

Formula honi fusc funtzioa ere esaten zaio [3]. Ohartu daitekeenez, errekurtsiboa da formula. 
Calkin eta Wilf-ek [4] honela interpretatzen dute a $(n+1)$ balioa: zenbat modu desberdinetan idatz daitekeen n zenbakia 2-ren berreturen baturak erabiliz, berretura bakoitza gehienez bi aldiz onartuta (hau da, adierazpide bitarrak gehienez onartzen duena baino aldi bat gehiago). Adibidez, $5=2^{2}+2^{0}=2^{1}+2^{1}+2^{0}$, eta $a(5+1)=2$. Egileek a $(n+1)$-ri n-ren adierazpide hiperbitarra esaten diote.

Lehmer-ek Stern-en ikerketa sakondu eta luzatu egin zuen propietate gehiago azalduz [5]. Gerora, denboran zehar gaur egun arte, hainbat ikertzailek segidaren propietateak sakontzen jarraitu dute, [6-9] besteak beste.

2.taula. Stern-en segida.

Segida honek garrantzia du hainbat zenbaki arrazionalen $\left(\mathbb{Q}^{+}\right)$zenbakitze-sistemaren oinarrian dagoelako. Esate baterako, Calkin-Wilf-en zenbakitze-sistema [3]: a (n) bada Stern-en segidaren n. gaia, a $(\mathrm{n}) / \mathrm{a}(\mathrm{n}+1)$ $(n>0)$ zatiki-segidak zenbaki arrazional positibo guztien segida osatzen $\mathrm{du}$, bat ere errepikatu gabe. Bantchev matematikari bulgariarrak dio [10] greziarren garaitik dela ezaguna sistema hau. Nonbait I. mendearen bukaera inguruko Nikomako Gerasakoak eta Theon Smyrnakoak aipatzen dute sistema hau; azken honek dio Eratostenes Zirenekoak, k.a. III. mendekoak, ezaguna zuela, eta beraz, asmatzailea k.a. Iv. mendekoa izan zitekeela.

Stern-en segida The On-Line Encyclopedia of Integer Sequences (OEIS)-en A002487 etiketaduna da [11]. Han ikus daitezke hainbat ikertzailek aurkitu dizkioten hainbat propietate, formula, konputazio-programa, eta beste zenbait segidarekin dituen loturak eta erreferentziak, bai eta beste zenbait problema matematikori buruzko iruzkinak ere. Webgunea etengabe ari da berritzen eta hazten.

Segidaren zenbatgarrena, $\mathrm{n}(>0)$, eta dagokion zenbakiaren tamaina, a (n), kontuan hartuz gero, segida irudikatu ere egin daiteke (ikus 1. irudia). Irudiak erakusten du segida zein errepikakorra den formaren aldetik, zatika, tamainaren aldetik ez bada ere.

Lan honetan azaltzen den problema da segidaren $\mathrm{n}$. gaiaren balioa (a $(n), n>0)$ ahalik eta azkarren kalkulatzea; hau da, definizio-formularen errekurtsibitatea arintzea. 

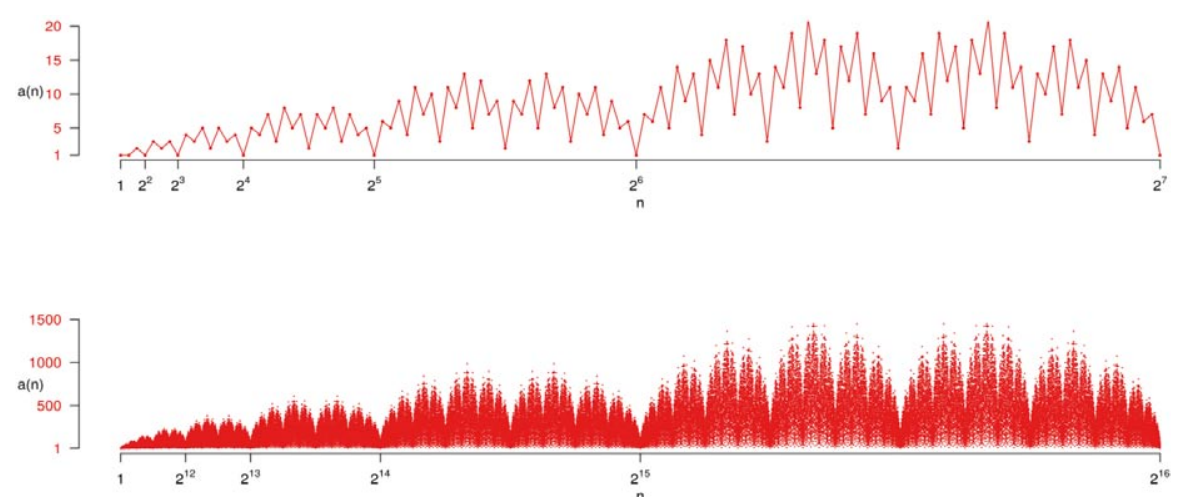

1. irudia. Stern-en segidaren bi irudikapen grafiko.

\section{STERN-EN SEGIDAREN PROPIETATE BERRI BAT}

Propietate berria enuntziatu aurretik n zenbaki arruntaren adierazpide mota bat aurkeztu behar da:

$$
\begin{aligned}
& \forall \mathrm{n}>0, \exists_{1} \mathrm{~m} \geq 0, \text { eta } \exists_{1} \mathrm{k} 0 \leq \mathrm{k}<2 \mathrm{~m}, \\
& \mathrm{n}=2^{\mathrm{m}}+\mathrm{k} \text { delarik. }
\end{aligned}
$$

Adierazpide hau egiazkoa da. Hori ikusteko nahikoa da infiniturantz doan $2^{\mathrm{m}}$ segida gorakorra kontuan hartzea eta $\mathrm{n}$ halako bi gairen artean kokatzea: $\exists_{1} \mathrm{~m} \geq 0,2^{\mathrm{m}} \leq \mathrm{n}<2^{\mathrm{m}+1}$. Orduan, nahikoa da $\mathrm{k}$ honela definitzea: $\mathrm{k}=\mathrm{n}-2^{\mathrm{m}}$. Hortaz, $0 \leq \mathrm{k}<2^{\mathrm{m}}$.

Adierazpide honek sistema bitarretik badu zertxobait, bai eta hamartarretik ere (mistoa, beraz).

Horrela, segidaren a $(\mathrm{n})$ balioak a $\left(2^{\mathrm{m}}+\mathrm{k}\right)$-ren bitartez azalduko dira. Adierazpide honen arabera, definizio-formula hauxe da:

$$
\begin{aligned}
& \mathrm{a}(1)=1, \text { eta } \forall \mathrm{m} \geq 0, \forall \mathrm{k} 0 \leq \mathrm{k}<2^{\mathrm{m}}: \\
& \mathrm{a}\left(2^{\mathrm{m}+1}+2 \mathrm{k} \quad\right)=\mathrm{a}\left(2^{\mathrm{m}}+\mathrm{k}\right) \\
& \mathrm{a}\left(2^{\mathrm{m}+1}+2 \mathrm{k}+1\right)=\mathrm{a}\left(2^{\mathrm{m}}+\mathrm{k}\right)+\mathrm{a}\left(2^{\mathrm{m}}+\mathrm{k}+1\right)
\end{aligned}
$$

2. taulako segidan a (n) balioak ezkerraldean pilatzen badira, egitura triangeluar bat osatzen da (ikus 3. taula). 
3. taula. Stern-en segidaren irudikapen triangeluarra.

$\begin{array}{lllllllllllllllllllllllllllllllllll}0 & 1 & 2 & 3 & 4 & 5 & 6 & 7 & 8 & 9 & 10 & 11 & 12 & 13 & 14 & 15 & 16 & 17 & 18 & 19 & 20 & 21 & 22 & 23 & 24 & 25 & 26 & 27 & 28 & 29 & 30 & 31\end{array}$

m $\quad 0 \quad 1$

$1 \quad 1 \quad 2$

$\begin{array}{llllll}2 & 1 & 3 & 2 & 3\end{array}$

$\begin{array}{llllllllll}3 & 1 & 4 & 3 & 5 & 2 & 5 & 3 & 4\end{array}$

$\begin{array}{llllllllllllllllllll}4 & 1 & 5 & 4 & 7 & 3 & 8 & 5 & 7 & 2 & 7 & 5 & 8 & 3 & 7 & 4 & 5\end{array}$

$\begin{array}{lllllllllllllllllllllllllllllllll}5 & 1 & 6 & 5 & 9 & 4 & 11 & 7 & 10 & 3 & 11 & 8 & 13 & 5 & 12 & 7 & 9 & 2 & 9 & 7 & 12 & 5 & 13 & 8 & 11 & 3 & 10 & 7 & 11 & 4 & 9 & 5 & 6\end{array}$

$\begin{array}{lllllllllllllllllllllllllllllllllll}6 & 1 & 7 & 6 & 11 & 5 & 14 & 9 & 13 & 4 & 15 & 11 & 18 & 7 & 17 & 10 & 13 & 3 & 14 & 11 & 19 & 8 & 21 & 13 & 18 & 5 & 17 & 12 & 19 & 7 & 16 & 9 & 11 \ldots\end{array}$

$\begin{array}{lllllllllllllllllllllllllllllllll}7 & 1 & 8 & 7 & 13 & 6 & 17 & 11 & 16 & 5 & 19 & 14 & 23 & 9 & 22 & 13 & 17 & 4 & 19 & 15 & 26 & 11 & 29 & 18 & 25 & 7 & 24 & 17 & 27 & 10 & 23 & 13 & 16\end{array}$

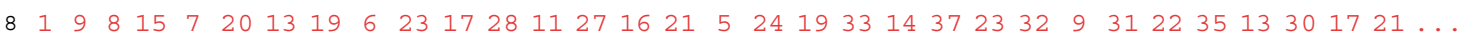

Modu honetan azalduta, segidaren propietate batzuk nabarmenak dira. Lerroka begiratuta:

$-\forall \mathrm{m} \geq 0,2^{\mathrm{m}}$ gai dago.

$-\forall \mathrm{m} \geq 0, a\left(2^{\mathrm{m}}\right)=1 ; a\left(2^{\mathrm{m}+1}+2^{\mathrm{m}}\right)=2 ; a\left(2^{\mathrm{m}}+1\right)=\mathrm{m}+1 ;$ $\mathrm{a}\left(2^{\mathrm{m}+1}-1\right)=\mathrm{m}+1$.

$-\forall \mathrm{m} \geq 0, \forall \mathrm{k} 0 \leq \mathrm{k}<2^{\mathrm{m}}, \mathrm{a}\left(2^{\mathrm{m}}+\mathrm{k}\right)=\mathrm{a}\left(2^{\mathrm{m}+1}-\mathrm{k}\right)$. (Simetria $)$.

Zutabeka begiratuta:

$-\forall \mathrm{m} \geq 0, \forall \mathrm{k} \quad 0 \leq \mathrm{k}<2^{\mathrm{m}}, a\left(2^{\mathrm{m}+1}+\mathrm{k}\right) \geq \mathrm{a}\left(2^{\mathrm{m}}+\mathrm{k}\right)$

Gainera, zutabe bakoitzean segida aritmetiko bat ikusten da, eta diferentzien segida Stern-en segida bera da (ikus 4. taula).

4. taula. 3 Taulako zutabeetako segida aritmetikoen diferentzien $(\delta)$ segida.

$\mathrm{k}$

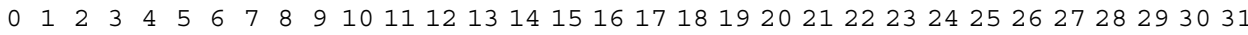
$\delta$

$\begin{array}{lllllllllllllllllllllllllllllllllllll}0 & 1 & 1 & 2 & 1 & 3 & 2 & 3 & 1 & 4 & 3 & 5 & 2 & 5 & 3 & 4 & 1 & 5 & 4 & 7 & 3 & 8 & 5 & 7 & 2 & 7 & 5 & 8 & 3 & 7 & 4 & 5 & \ldots\end{array}$

Aurkitutako erlazio hau honela zehaztu daiteke:

$$
\begin{aligned}
& a(0)=0, \text { eta } \forall m \geq 0, \quad 0 \leq k<2^{m}: \\
& a\left(2^{m+1}+k\right)-a\left(2^{m}+k\right)=a(k)
\end{aligned}
$$

Ohar daitekeenez, segidari beste gai bat gehitu behar izan zaio: $a(0)=0,2$. taulan agertzen ez dena. 
Propietate berria ez da hain nabarmena, baina 3. taulako zutabe bakoitzeko segida aritmetikoetan oinarritzen da, eta honela enuntzia daiteke:

$$
\begin{aligned}
& \mathrm{a}(0)=0, \\
& \forall \mathrm{m} \geq 0 \mathrm{a}\left(2^{\mathrm{m}}\right)=1, \forall \mathrm{m} \geq 1 \mathrm{a}\left(2^{\mathrm{m}}+1\right)=\mathrm{m}+1, \\
& \forall \mathrm{m} \geq 2,0<\mathrm{m}^{\prime}<\mathrm{m}, 0 \leq \mathrm{k}^{\prime}<2^{\mathrm{m}^{\prime}}: \\
& \mathrm{a}\left(2^{\mathrm{m}}+2^{\mathrm{m}^{\prime}}+\mathrm{k}^{\prime}\right)=\mathrm{a}\left(2^{\mathrm{m}^{\prime}}+\mathrm{k}^{\prime}\right) \cdot\left(\mathrm{m}-\left(\mathrm{m}^{\prime}-1\right)\right)-\mathrm{a}\left(\mathrm{k}^{\prime}\right)
\end{aligned}
$$

Bi formula errekurtsiboak alderatzean, ikusten da definizio-formularen errekurtsibitatea 3. taularen maila batetik ondoz ondoko mailara igarotzean datzala $((m+1)$-tik m-ra), eta propietate berriarenean, aldiz, maila batetik aurreko beste maila batera igarotzean (m-tik $\left.\mathrm{m}^{\prime}-\mathrm{ra}, \mathrm{m}^{\prime}<\mathrm{m}\right)$; hau da, propietate berriaren errekurtsibitatean jauzi handiagoak gerta daitezke, eta hortik errekurtsibitatea azkarragoa izatea espero liteke.

\subsection{Propietatearen sorrera}

Irudikapen triangeluarrari begira (ikus 3. taula), zutabeetako segida aritmetikoak deskriba daitezke m-ren arabera (ikus 5. taula).

Zutabe guztietan m-ren biderkagaia dagokion segida aritmetikoaren diferentzia da (ikus 4. taula), noski. Lehenengo bi lerroak bereziak dira gainerakoen ondoan:

$$
\begin{aligned}
& \forall \mathrm{m} \geq 0 \mathrm{a}\left(2^{\mathrm{m}}\right)=1 \\
& \forall \mathrm{m} \geq 1 \mathrm{a}\left(2^{\mathrm{m}}+1\right)=\mathrm{m}+1
\end{aligned}
$$

Gainerako kasuetan gai kentzailea ere segidarekin berarekin dago lotuta. Areago, berdintza guztien patroia, induktiboki burutua, formula honen bitartez adieraz daiteke:

$$
\begin{aligned}
& \forall \mathrm{m} \geq 2,0<\mathrm{m}^{\prime}<\mathrm{m}, 0 \leq \mathrm{k}^{\prime}<2^{\mathrm{m}^{\prime}}: \\
& \mathrm{a}\left(2^{\mathrm{m}}+2^{\mathrm{m}^{\prime}}+\mathrm{k}^{\prime}\right)=\mathrm{a}\left(2^{\mathrm{m}^{\prime}}+\mathrm{k}^{\prime}\right) \cdot\left(\mathrm{m}-\left(\mathrm{m}^{\prime}-1\right)\right)-\mathrm{a}\left(\mathrm{k}^{\prime}\right), \\
& \mathrm{k}^{\prime}=0 \text { kasurako, } \mathrm{a}(0)=0 \text { beharrezkoa da. }
\end{aligned}
$$

Propietate hau, beraz, esperimentalki lortu da. 
5. taula. 3. taulako zutabeetako segida aritmetikoak.
$\mathrm{a}\left(2^{\mathrm{m}}\right)=\mathrm{a}\left(2^{\mathrm{m}} \quad\right)=1 \quad=0(\mathrm{~m} \quad)+1, \mathrm{~m} \geq 0$.
$\mathrm{a}\left(2^{\mathrm{m}}+1\right)=\mathrm{a}\left(2^{\mathrm{m}}+2^{0} \quad\right)=\mathrm{m}+1=1(\mathrm{~m}+1), \mathrm{m} \geq 1$.
$\mathrm{a}\left(2^{\mathrm{m}}+2\right)=\mathrm{a}\left(2^{\mathrm{m}}+2^{1} \quad\right)=\mathrm{m}=1(\mathrm{~m}), \mathrm{m} \geq 2$.
$\mathrm{a}\left(2^{\mathrm{m}}+3\right)=\mathrm{a}\left(2^{\mathrm{m}}+2^{1}+1\right)=2 \mathrm{~m}-1=2(\mathrm{~m} \quad)-1, \mathrm{~m} \geq 2$.
$\mathrm{a}\left(2^{\mathrm{m}}+4\right)=\mathrm{a}\left(2^{\mathrm{m}}+2^{2}+\right)=\mathrm{m}-1=1(\mathrm{~m}-1), \mathrm{m} \geq 3$.
$\mathrm{a}\left(2^{\mathrm{m}}+5\right)=\mathrm{a}\left(2^{\mathrm{m}}+2^{2}+1\right)=3 \mathrm{~m}-4=3(\mathrm{~m}-1)-1, \mathrm{~m} \geq 3$.
$a\left(2^{m}+6\right)=a\left(2^{m}+2^{2}+2\right)=2 m-3=2(m-1)-1, m \geq 3$.
$\mathrm{a}\left(2^{\mathrm{m}}+7\right)=\mathrm{a}\left(2^{\mathrm{m}}+2^{2}+3\right)=3 \mathrm{~m}-5=3(\mathrm{~m}-1)-2, \mathrm{~m} \geq 3$.
$\mathrm{a}\left(2^{\mathrm{m}}+8\right)=\mathrm{a}\left(2^{\mathrm{m}}+2^{3} \quad\right)=\mathrm{m}-2=1(\mathrm{~m}-2), \mathrm{m} \geq 4$.
$\mathrm{a}\left(2^{\mathrm{m}}+9\right)=\mathrm{a}\left(2^{\mathrm{m}}+2^{3}+1\right)=4 \mathrm{~m}-9=4(\mathrm{~m}-2)-1, \mathrm{~m} \geq 4$.
$\mathrm{a}\left(2^{\mathrm{m}}+10\right)=\mathrm{a}\left(2^{\mathrm{m}}+2^{3}+2\right)=3 \mathrm{~m}-7=3(\mathrm{~m}-2)-1, \mathrm{~m} \geq 4$.
$\mathrm{a}\left(2^{\mathrm{m}}+11\right)=\mathrm{a}\left(2^{\mathrm{m}}+2^{3}+3\right)=5 \mathrm{~m}-12=5(\mathrm{~m}-2)-2, \mathrm{~m} \geq 4$.
$a\left(2^{m}+12\right)=a\left(2^{m}+2^{3}+4\right)=2 m-5=2(m-2)-1, m \geq 4$.
$\mathrm{a}\left(2^{\mathrm{m}}+13\right)=\mathrm{a}\left(2^{\mathrm{m}}+2^{3}+5\right)=5 \mathrm{~m}-13=5(\mathrm{~m}-2)-3, \mathrm{~m} \geq 4$.
$\mathrm{a}\left(2^{\mathrm{m}}+14\right)=\mathrm{a}\left(2^{\mathrm{m}}+2^{3}+6\right)=3 \mathrm{~m}-8=3(\mathrm{~m}-2)-2, \mathrm{~m} \geq 4$.
$\mathrm{a}\left(2^{\mathrm{m}}+15\right)=\mathrm{a}\left(2^{\mathrm{m}}+2^{3}+7\right)=4 \mathrm{~m}-11=4(\mathrm{~m}-2)-3, \mathrm{~m} \geq 4$.
$\mathrm{a}\left(2^{\mathrm{m}}+16\right)=\mathrm{a}\left(2^{\mathrm{m}}+2^{4} \quad\right)=\mathrm{m}-3=1(\mathrm{~m}-3), \mathrm{m} \geq 5$.
$a\left(2^{m}+17\right)=a\left(2^{m}+2^{4}+1\right)=5 m-16=5(m-3)-1, m \geq 5$.
$\mathrm{a}\left(2^{\mathrm{m}}+18\right)=\mathrm{a}\left(2^{\mathrm{m}}+2^{4}+2\right)=4 \mathrm{~m}-13=4(\mathrm{~m}-3)-1, \mathrm{~m} \geq 5$.
$\mathrm{a}\left(2^{\mathrm{m}}+19\right)=\mathrm{a}\left(2^{\mathrm{m}}+2^{4}+3\right)=7 \mathrm{~m}-23=7(\mathrm{~m}-3)-2, \mathrm{~m} \geq 5$.
$a\left(2^{m}+20\right)=a\left(2^{m}+2^{4}+4\right)=3 m-10=3(m-3)-1, m \geq 5$.
$a\left(2^{m}+21\right)=a\left(2^{m}+2^{4}+5\right)=8 m-27=8(m-3)-3, m \geq 5$.
$a\left(2^{m}+22\right)=a\left(2^{m}+2^{4}+6\right)=5 m-17=5(m-3)-2, m \geq 5$.
$a\left(2^{m}+23\right)=a\left(2^{m}+2^{4}+7\right)=7 m-24=7(m-3)-3, m \geq 5$.
$\mathrm{a}\left(2^{\mathrm{m}}+24\right)=\mathrm{a}\left(2^{\mathrm{m}}+2^{4}+8\right)=2 \mathrm{~m}-7=2(\mathrm{~m}-3)-1, \mathrm{~m} \geq 5$.
$\mathrm{a}\left(2^{\mathrm{m}}+25\right)=\mathrm{a}\left(2^{\mathrm{m}}+2^{4}+9\right)=7 \mathrm{~m}-25=7(\mathrm{~m}-3)-4, \mathrm{~m} \geq 5$.
$a\left(2^{m}+26\right)=a\left(2^{m}+2^{4}+10\right)=5 m-18=5(m-3)-3, m \geq 5$.
$a\left(2^{m}+27\right)=a\left(2^{m}+2^{4}+11\right)=8 m-29=8(m-3)-5, m \geq 5$.
$a\left(2^{m}+28\right)=a\left(2^{m}+2^{4}+12\right)=3 m-11=3(m-3)-2, m \geq 5$.
$a\left(2^{m}+29\right)=a\left(2^{m}+2^{4}+13\right)=7 m-26=7(m-3)-5, m \geq 5$.
$a\left(2^{m}+30\right)=a\left(2^{m}+2^{4}+14\right)=4 m-15=4(m-3)-3, m \geq 5$.
$\mathrm{a}\left(2^{\mathrm{m}}+31\right)=\mathrm{a}\left(2^{\mathrm{m}}+2^{4}+15\right)=5 \mathrm{~m}-19=5(\mathrm{~m}-3)-4, \mathrm{~m} \geq 5$.

\subsection{Propietatearen froga matematikoa}

Propietatearen egiazkotasuna m parametroaren arabera froga daiteke. Funtsean, definizio-formulazioa atzera-aurrera erabiltzen da, baita propietatearen beraren formulazioa ere indukzio matematikoa erabiliz. 
1. $\forall \mathrm{m} \geq 0, \mathrm{a}\left(2^{\mathrm{m}}\right)=1$

$\mathrm{a}\left(2^{\mathrm{m}+1}\right)=\mathrm{a}\left(2\left(2^{\mathrm{m}}\right)\right)=\mathrm{a}\left(2^{\mathrm{m}}\right) ; \quad\left\{\mathrm{a}\left(2^{\mathrm{m}}\right)\right\}_{\mathrm{m} \geq 0} \quad$ segida konstantea da, eta $\mathrm{a}\left(2^{0}\right)=\mathrm{a}(1)=1$.

2. $\forall \mathrm{m} \geq 1 \mathrm{a}\left(2^{\mathrm{m}}+1\right)=\mathrm{m}+1$

Oinarrizko kasua: $m=1$,

$a\left(2^{1}+1\right)=a(2 \cdot 1+1)=a(1)+a(2)=a\left(2^{0}\right)+a\left(2^{1}\right)=1+1$.

Indukzio-hipotesia: $\mathrm{m}>1$,

$$
\forall m^{\prime} 0 \leq m^{\prime} \leq m, a\left(2^{m^{\prime}}+1\right)=m^{\prime}+1 \text {. }
$$

Indukzio-urratsa: $(m+1)$.

$\mathrm{a}\left(2^{\mathrm{m}+1}+1\right)=\mathrm{a}\left(2 \cdot 2^{\mathrm{m}}+1\right)=\mathrm{a}\left(2^{\mathrm{m}}\right)+\mathrm{a}\left(2^{\mathrm{m}}+1\right)=1+(\mathrm{m}+1)=(\mathrm{m}+1)+1$.

3. $\mathrm{a}(0)=0, \forall \mathrm{m} \geq 2,0<\mathrm{m}^{\prime}<\mathrm{m}, 0 \leq \mathrm{k}^{\prime}<2^{\mathrm{m}^{\prime}}$ :

$\mathrm{a}\left(2^{\mathrm{m}}+2^{\mathrm{m}^{\prime}}+\mathrm{k}^{\prime}\right)=\mathrm{a}\left(2^{\mathrm{m}^{\prime}}+\mathrm{k}^{\prime}\right) \cdot\left(\mathrm{m}-\mathrm{m}^{\prime}+1\right)-\mathrm{a}\left(\mathrm{k}^{\prime}\right)$

$\mathrm{k}^{\prime}=0$ kasurako, $\mathrm{a}(0)=0$ beharrezkoa da.

Oinarrizko kasua: $\mathrm{m}=2$; ezinbestean da $\mathrm{m}^{\prime}=1$, eta beraz bi kasu daude: $\mathrm{k}^{\prime}=0$ eta $\mathrm{k}^{\prime}=1$.

$* \mathrm{k}^{\prime}=0$;

$$
\begin{aligned}
& \begin{aligned}
\mathrm{a}\left(2^{2}\right. & \left.+2^{1}+0\right)=\mathrm{a}(6)=\mathrm{a}(2 \cdot 3)=\mathrm{a}(3)=\mathrm{a}(2 \cdot 1+1)= \\
& =\mathrm{a}(1)+\mathrm{a}(2)=1+1=\mathrm{a}(1) \cdot 2-\mathrm{a}(0)= \\
& =\mathrm{a}\left(2^{0}+0\right) \cdot(2-1+1)-\mathrm{a}(0) .
\end{aligned} \\
& \text { * } \mathrm{k}^{\prime}=1 ; \\
& \mathrm{a}\left(2^{2}+2^{1}+1\right)=\mathrm{a}(7)=\mathrm{a}(2 \cdot 3+1)=\mathrm{a}(3)+\mathrm{a}(4)= \\
& =2+1=\mathrm{a}(3) \cdot 2-1=\mathrm{a}\left(2^{1}+1\right) \cdot(2-1+1)-\mathrm{a}(1) .
\end{aligned}
$$

Indukzio-hipotesia: izan bedi $\mathrm{m}>2$, eta izan bitez $0<\mathrm{m}^{\prime}<\mathrm{m}$, eta $\mathrm{k}^{\prime} 0 \leq \mathrm{k}^{\prime}<2^{\mathrm{m}^{\prime}}$

$$
a\left(2^{m}+2^{m^{\prime}}+k^{\prime}\right)=a\left(2^{m^{\prime}}+k^{\prime}\right) \cdot\left(m-m^{\prime}+1\right)-a\left(k^{\prime}\right)
$$

Indukzio-urratsa: $(m+1)$. Izan bitez $\mathrm{m}^{\prime}, 0<\mathrm{m}^{\prime}<(m+1)$, eta $\mathrm{k}^{\prime} 0 \leq \mathrm{k}^{\prime}<2^{\mathrm{m}^{\prime}}$.

Horretarako, $\mathrm{k}^{\prime}$-ren bakoiti-bikoiti izaera bereizi behar da, eta definizio-formula proposatutako formularekin konbinatu: 
* $\mathrm{k}^{\prime}$ bikoitia: $\exists_{1} \mathrm{k} \geq 0, \mathrm{k}^{\prime}=2 \mathrm{k} . \quad$ Beraz, $0 \leq \mathrm{k}<2^{\mathrm{m}^{\prime}-1}$.

$$
\begin{aligned}
\mathrm{a}\left(2^{\mathrm{m}+1}\right. & \left.+2^{\mathrm{m}^{\prime}}+\mathrm{k}^{\prime}\right)=\mathrm{a}\left(2\left(2^{\mathrm{m}}+2^{\mathrm{m}^{\prime}-1}+\mathrm{k}\right)\right)=\mathrm{a}\left(2^{\mathrm{m}}+2^{\mathrm{m}^{\prime}-1}+\mathrm{k}\right)= \\
& =\mathrm{a}\left(2^{\mathrm{m}^{\prime}-1}+\mathrm{k}\right) \cdot\left(\mathrm{m}-\left(\mathrm{m}^{\prime}-1\right)+1\right)-\mathrm{a}(\mathrm{k})= \\
& =\mathrm{a}\left(2\left(2^{\mathrm{m}^{\prime}-1}+\mathrm{k}\right)\right) \cdot\left(\mathrm{m}-\left(\mathrm{m}^{\prime}-1\right)+1\right)-\mathrm{a}(2 \mathrm{k})= \\
& =\mathrm{a}\left(2^{\mathrm{m}^{\prime}}+2 \mathrm{k}\right) \cdot\left(\mathrm{m}-\left(\mathrm{m}^{\prime}-1\right)+1\right)-\mathrm{a}(2 \mathrm{k})= \\
& =\mathrm{a}\left(2^{\mathrm{m}^{\prime}}+\mathrm{k}^{\prime}\right) \cdot\left((\mathrm{m}+1)-\mathrm{m}^{\prime}+1\right)-\mathrm{a}\left(\mathrm{k}^{\prime}\right) .
\end{aligned}
$$

* $\mathrm{k}^{\prime}$ bakoitia: $\exists_{1} \mathrm{k} \geq 0, \mathrm{k}^{\prime}=2 \mathrm{k}+1$.

Beraz, $0 \leq \mathrm{k}<2^{\mathrm{m}^{\prime}-1}-1 / 2<2^{\mathrm{m}^{\prime}-1}$.

Parekoa den desberdintza honetan $\left(1 \leq k+1<2^{m^{\prime}-1}+1 / 2\right)$ bi kasu bereizten dira: $1 \leq \mathrm{k}+1<2^{\mathrm{m}^{\prime}-1}$ eta $\mathrm{k}+1=2^{\mathrm{m}^{\prime}-1}$.

$$
\begin{aligned}
& \text { * } 1 \leq \mathrm{k}+1<2^{\mathrm{m}^{\prime}-1} \text { : } \\
& \mathrm{a}\left(2^{\mathrm{m}+1}+2^{\mathrm{m}^{\prime}}+\mathrm{k}^{\prime}\right)=\mathrm{a}\left(2\left(2^{\mathrm{m}}+2^{\mathrm{m}^{\prime}-1}+\mathrm{k}\right)+1\right)= \\
& =\mathrm{a}\left(2^{\mathrm{m}}+2^{\mathrm{m}^{\prime}-1}+\mathrm{k}\right)+\mathrm{a}\left(2^{\mathrm{m}}+2^{\mathrm{m}^{\prime}-1}+\mathrm{k}+1\right)= \\
& =\mathrm{a}\left(2^{\mathrm{m}^{\prime}-1}+\mathrm{k}\right) \cdot\left(\mathrm{m}-\left(\mathrm{m}^{\prime}-1\right)+1\right)-\mathrm{a}(\mathrm{k})+ \\
& \mathrm{a}\left(2^{\mathrm{m}^{\prime}-1}+\mathrm{k}+1\right) \cdot\left(\mathrm{m}-\left(\mathrm{m}^{\prime}-1\right)+1\right)-\mathrm{a}(\mathrm{k}+1)= \\
& =\left(\mathrm{a}\left(2^{\mathrm{m}^{\prime}-1}+\mathrm{k}\right)+\mathrm{a}\left(2^{\mathrm{m}^{\prime}-1}+\mathrm{k}+1\right)\right) \cdot\left(\mathrm{m}-\left(\mathrm{m}^{\prime}-1\right)+1\right)- \\
& -(\mathrm{a}(\mathrm{k})+\mathrm{a}(\mathrm{k}+1))= \\
& =a\left(2\left(2^{m^{\prime}-1}+k\right)+1\right) \cdot\left((m+1)-m^{\prime}+1\right)-a(2 k+1)= \\
& =\mathrm{a}\left(2^{\mathrm{m}^{\prime}}+2 \mathrm{k}+1\right) \cdot\left((\mathrm{m}+1)-\mathrm{m}^{\prime}+1\right)-\mathrm{a}(2 \mathrm{k}+1)= \\
& =a\left(2^{m^{\prime}}+k^{\prime}\right) \cdot\left((m+1)-m^{\prime}+1\right)-a\left(k^{\prime}\right) \text {. } \\
& * \mathrm{k}+1=2^{\mathrm{m}^{\prime}-1} \text { Beraz, } \mathrm{k}^{\prime}=2 \mathrm{k}+1=2^{\mathrm{m}^{\prime}}-1 \text {. } \\
& \mathrm{a}\left(2^{\mathrm{m}+1}+2^{\mathrm{m}^{\prime}}+\mathrm{k}^{\prime}\right)=\mathrm{a}\left(2^{\mathrm{m}+1}+2^{\mathrm{m}^{\prime}}+2^{\mathrm{m}^{\prime}}-1\right)= \\
& =a\left(2^{m+1}+2^{m^{\prime}+1}-1\right)=a\left(2\left(2^{m}+2^{m^{\prime}}-1\right)+1\right)= \\
& =\mathrm{a}\left(2^{\mathrm{m}}+2^{\mathrm{m}^{\prime}}-1\right) \quad+\mathrm{a}\left(2^{\mathrm{m}}+2^{\mathrm{m}^{\prime}}\right)= \\
& =a\left(2^{m}+2^{m^{\prime}}-1+2^{m^{\prime}-1}-1\right)+a\left(2^{m^{\prime}}\left(2^{m-m^{\prime}}+1\right)\right)= \\
& =a\left(2^{m^{\prime}}-1\right) \cdot\left(m-\left(m^{\prime}-1\right)+1\right)-a\left(2^{m^{\prime}-1}-1\right)+ \\
& +\left(m-m^{\prime}+1\right)= \\
& =\left(a\left(2^{m^{\prime}}-1\right)+1\right) \cdot\left((m+1)-m^{\prime}+1\right)- \\
& -\left(\mathrm{a}\left(2^{\mathrm{m}^{\prime}-1}-1\right)+1\right)= \\
& =\left(a\left(2^{m^{\prime}}-1\right)+a\left(2^{m^{\prime}}\right)\right) \cdot\left((m+1)-m^{\prime}+1\right)- \\
& -\left(a\left(2^{m^{\prime}-1}-1\right)+a\left(2^{m^{\prime}-1}\right)\right)= \\
& =a\left(2^{m^{\prime}+1}-1\right) \cdot\left((m+1)-m^{\prime}+1\right)-a\left(2^{m^{\prime}}-1\right)= \\
& =a\left(2^{m^{\prime}}+k^{\prime}\right) \cdot\left((m+1)-m^{\prime}+1\right)-a\left(k^{\prime}\right) \text {. }
\end{aligned}
$$




\subsection{Propietatearen garapena}

Aurreko formula garatuz gero, errekurtsiboki (goitik behera, top-down, $\mathrm{m}$ handitik txikira), a (n) -ren balioa kalkulatzeko nahikoak dira n-ren adierazpide bitarra eta segidaren hasierako a $(0)=0, a(1)=1$ eta $a(2)=1$ balioak.

Adibidez:

$$
\mathrm{n}=91=2^{6}+2^{4}+2^{3}+2^{1}+2^{0} .
$$

Haren adierazpide bitarra: $91_{10} \equiv 1011011_{2}$.

5 '1'-eko dauzka: $b_{6}=b_{4}=b_{3}=b_{1}=b_{0}=1$.

$$
\begin{aligned}
\mathrm{a}(91) & =\mathrm{a}\left(2^{6}+2^{4}+11\right)=\mathrm{a}\left(2^{4}+11\right) \cdot(6-4+1)-\mathrm{a}(11)= \\
& =\mathrm{a}\left(2^{4}+11\right) \cdot \mathrm{C}_{4}-\mathrm{a}(11)=\mathrm{a}(27) \cdot \mathrm{C}_{4}-\mathrm{a}(11), \\
& \left.\mathrm{C}_{4}=(6-4+1)\right)=3 \text { delarik. }
\end{aligned}
$$$$
a(27)=a\left(2^{4}+2^{3}+3\right)=a\left(2^{3}+3\right) \cdot(4-3+1)-a(3)=
$$$$
=a\left(2^{3}+3\right) \cdot c_{3}-a(3)=a(11) \cdot c_{3}-a(3) \text {, }
$$$$
C_{3}=(4-3+1)=2 \text { delarik. }
$$

$$
\begin{aligned}
\mathrm{a}(11) & =\mathrm{a}\left(2^{3}+2^{1}+1\right)=\mathrm{a}\left(2^{1}+1\right) \cdot(3-1+1)-\mathrm{a}(1)= \\
& =\mathrm{a}\left(2^{1}+1\right) \cdot \mathrm{c}_{2}-\mathrm{a}(1)=\mathrm{a}(3) \cdot \mathrm{c}_{2}-\mathrm{a}(1), \\
& \mathrm{C}_{2}=(3-1+1)=3 \text { delarik. }
\end{aligned}
$$

$$
\begin{aligned}
\mathrm{a}(3)= & \mathrm{a}\left(2^{1}+2^{0}+0\right)=\mathrm{a}\left(2^{0}+0\right) \cdot(1-(0-1))-\mathrm{a}(0)= \\
& \left.=\mathrm{a}\left(2^{0}+0\right) \cdot \mathrm{c}_{1}-\mathrm{a}(0)=\mathrm{a}(1) \cdot \mathrm{c}_{1}-\mathrm{a} 0\right), \\
& \mathrm{C}_{1}=(1-0+1)=2 \text { delarik. }
\end{aligned}
$$

Ohartu daitekeenez, $\mathrm{a}(0)=0$ eta $\mathrm{a}(1)=1$ beharrezkoak dira $a(3)=2$ izan dadin.

Beraz, 91-ren adierazpide bitarrean oinarritzen diren $c_{j}(1 \leq j<5)$ biderkagaiak kalkulatuz, eta haiek egokiro konbinatuz gero (behetik gora, bottom-up), nahikoa da a (91) -ren balioa emateko.

Biderkagaiak kalkulatzeko, adierazpide bitarraren ' 1 'ekoen arteko jauziak hartzen dira kontuan.

Are gehiago, $\forall m \geq 0,2^{m} \cdot 91$ zenbakien $C_{j}(1 \leq j<5)$ biderkagaiak 91-renak berberak dira. Esaterako,

$$
\begin{gathered}
\mathrm{n}=728=2^{9}+2^{7}+2^{6}+2^{4}+2^{3}\left(728_{10} \equiv 1011011000_{2}\right) ; \\
\mathrm{a}(728)=\mathrm{a}\left(2^{3} \cdot 91\right)=\mathrm{a}(91) .
\end{gathered}
$$

Adierazpide bitarraren azken '0'ek ez dute parte hartzen biderkagaien kalkuluetan. 
Orokorrean:

Izan bedi $\mathrm{n} \in \mathrm{N}$, eta $\mathrm{b}_{\mathrm{m}} \mathrm{b}_{\mathrm{m}-1} \ldots \mathrm{b}_{1} \mathrm{~b}_{0}$ haren adierazpide bitar hau:

$$
\begin{aligned}
& \mathrm{n}=\mathrm{b}_{\mathrm{m}} 2^{\mathrm{m}}+\mathrm{b}_{\mathrm{m}-1} 2^{\mathrm{m}-1}+\ldots+\mathrm{b}_{1} 2^{1}+\mathrm{b}_{0} 2^{0}, \\
& \forall i \quad 0 \leq \mathrm{i}<\mathrm{m}, \mathrm{b}_{\mathrm{i}} \in\{0,1\}, \mathrm{b}_{\mathrm{m}}=1 .
\end{aligned}
$$

Izan bedi $\mathrm{b}=\mathrm{b}_{0}+\mathrm{b}_{1}+\ldots+\mathrm{b}_{\mathrm{m}-1}+\mathrm{b}_{\mathrm{m}}$, ' 1 '-eko balioa duten digitu bitarren kopurua $(\mathrm{b} \geq 1)$.

Izan bedi $\left\{m_{j}: j=1,2, \ldots, \quad b\right\}$ ' 1 '-eko balioa duten digitu bitarren azpiindize-multzoa; hau da:

$$
\mathrm{b}_{\mathrm{mj}}=1,1 \leq j \leq \mathrm{b} \text {, eta } 0 \leq \mathrm{m}_{1} \leq \mathrm{m}_{2} \leq \ldots \leq \mathrm{m}_{\mathrm{b}} \equiv \mathrm{m} .
$$

Biderkagai hauek n-ren adierazpide bitarra jartzen dute jokoan.

$\mathrm{b}=1$ baldin bada (' 1 '-eko bakarra), orduan izan bedi $\mathrm{c} 1=1$ $(\forall \mathrm{m} \geq 0, \mathrm{a}(2 \mathrm{~m})=1$ delako), eta bestela: $\forall j 1 \leq j<\mathrm{b}$, $c j=m j+1-m j+1$.

Biderkagai hauek ' 1 '-ekoen artean dauden jauziak adierazten dituzte.

Izan bedi f burututako formula errekurtsibo berria islatzen duen funtzio hau:

$$
\begin{aligned}
& f(0)=0(a(0)=0 \text { delako }), \\
& f(1)=1\left(a\left(2^{m}\right)=1 \text { delako }\right), \\
& \forall j \geq 0,1<j \leq b, f(j)=c_{j-1} \cdot f(j-1)-f(j-2) .
\end{aligned}
$$

Orduan: $a(n)=f(b)$.

f funtzioaren balio esplizituak hauek dira:

$$
\begin{aligned}
f(0)= & 0 \\
f(1)= & 1 \\
f(2)= & C_{1} \cdot 1-0=C_{1} \\
f(3)= & C_{1} \cdot C_{2}-1 \\
f(4)= & C_{1} \cdot C_{2} \cdot C_{3}-C_{3}-C_{1} \\
f(5)= & C_{1} \cdot C_{2} \cdot C_{3} \cdot C_{4}-C_{1} \cdot C_{2}-C_{1} \cdot C_{4}-C_{3} \cdot C_{4}+1 \\
f(6)= & C_{1} \cdot C_{2} \cdot C_{3} \cdot C_{4} \cdot C_{5}-C_{1} \cdot C_{2} \cdot C_{3}-C_{1} \cdot C_{2} \cdot C_{5}-C_{1} \cdot C_{4} \cdot C_{5}- \\
& -C_{3} \cdot C_{4} \cdot C_{5}+C_{1}+C_{3}+C_{5}
\end{aligned}
$$


Adibidea:

$$
\begin{aligned}
& \left.\mathrm{a}(91)=\mathrm{a}\left(2^{6}+27\right)=19 \text { (ikus 3. taulan } \mathrm{m}=6, \mathrm{k}=27\right) . \\
& \mathrm{a}\left(91_{10}\right)=\mathrm{a}\left(1011011_{2}\right) \\
& \mathrm{b}_{0}=\mathrm{b}_{1}=\mathrm{b}_{3}=\mathrm{b}_{4}=\mathrm{b}_{6}=1 ; \mathrm{b}=5 \\
& \mathrm{~m}_{1}=0, \mathrm{~m}_{2}=1, \mathrm{~m}_{3}=3, \mathrm{~m}_{4}=4, \mathrm{~m}_{5}=6 \\
& \mathrm{C}_{1}=2, \mathrm{C}_{2}=3, \mathrm{C}_{3}=2, \mathrm{C}_{4}=3 \\
& \mathrm{f}(0)=0, \mathrm{f}(1)=1, \mathrm{f}(2)=2, \mathrm{f}(3)=5, \mathrm{f}(4)=8, \\
& \mathrm{a}(91)=\mathrm{f}(5)=19 .
\end{aligned}
$$

\subsection{Propietatea garatu ondorengo kalkulua}

Hauxe da propietate berria islatzen duen $\mathrm{f}$ funtzioaren transkripzio bat $\boldsymbol{R}$ lengoaian [12]:

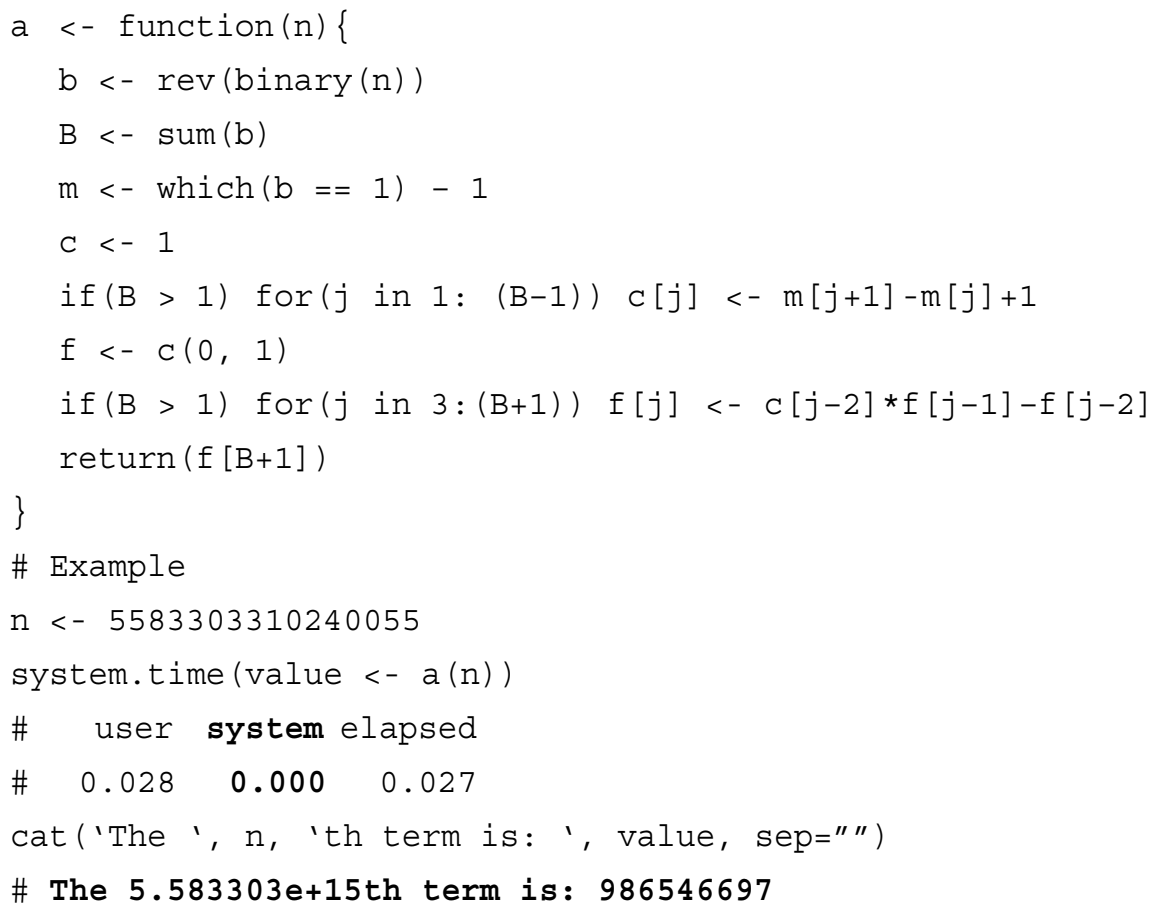

binary () funtzioak zenbaki arrunt bat adierazpide bitar bihurtzen du. $\boldsymbol{R}$-k badu berezko funtzio bat horretarako, intToBits (), baina zenbakiek $2^{32}$ baino txikiagoak behar dute izan. Hemen erabili den binary() funtzioa Crawley-ren izen bereko funtzioan dago oinarrituta [13]: 


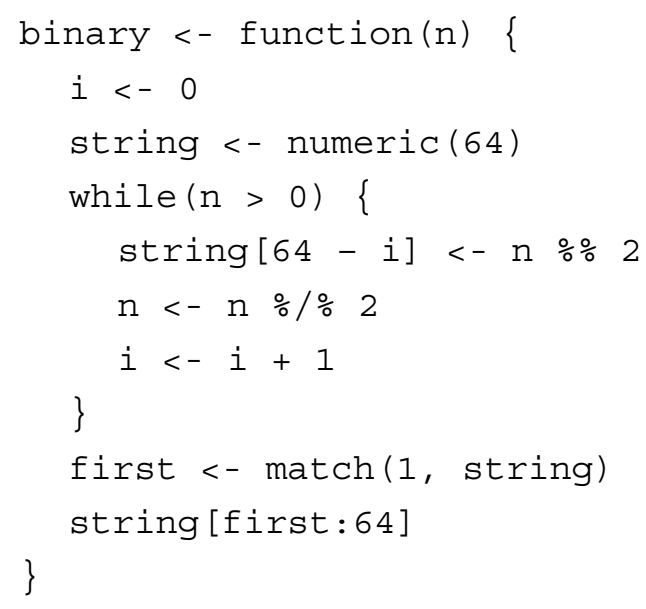

Propietate berrian oinarritutako algoritmoaren azkartasunaz jabetzearren, hasieran emandako definizio-formula errekurtsiboarekin alderatu daiteke:

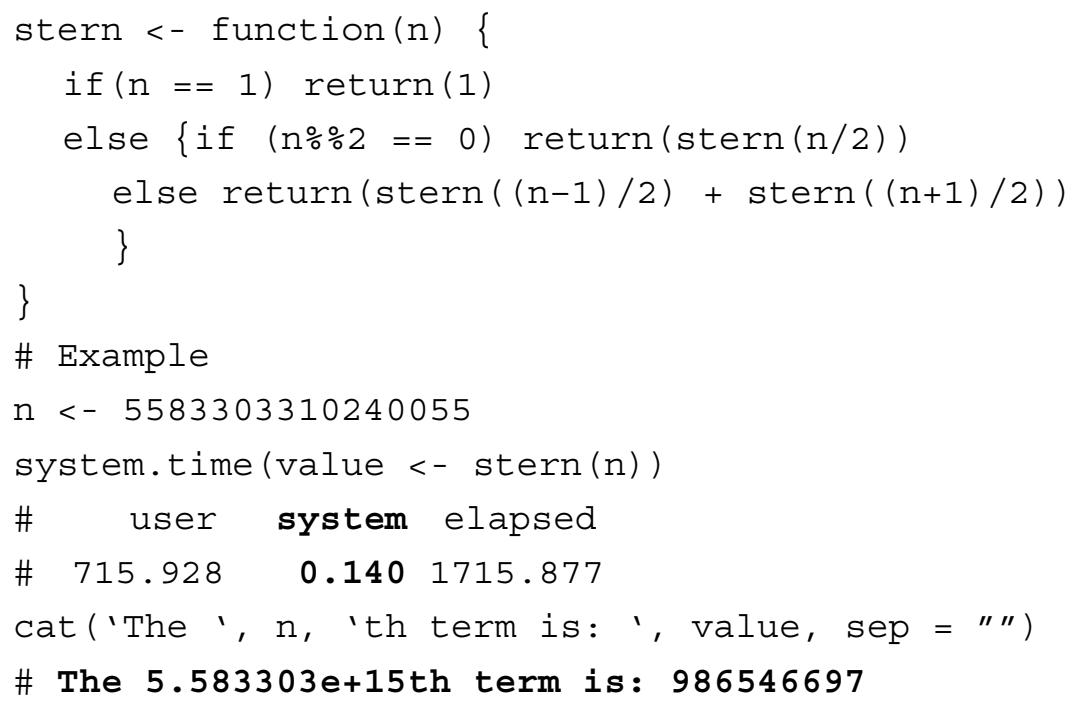

Propietate berrian oinarritutako algoritmoa dezente azkarragoa gertatu da definizio-formulan oinarritutakoa baino.

Bi algoritmoak exekutatzean erabilitako konputagailuaren ezaugarriak hauek dira: Memoria 15,5 GiB ; Prozesadorea Intel ${ }^{\circledR}$ Core $^{\mathrm{TM}}$ i7-3770 CPU @ 3.40GHz ×8; Grafikoak Intel@lvybridge Desktop ; SE mota 64bit; Diskoa 968,1 GB. 


\section{ONDORIOAK}

Stern-en segidaren propietate berri bat esperimentalki induzitu da, eta matematikoki haren egiazkotasuna frogatu. Formula horretan oinarrituz, segidaren edozein tokitako balioa kalkulatu daiteke, eta eraikitako algoritmoa konputazionalki azkarragoa da definizio-formulan oinarritutakoa baino. Algoritmo hori n-ren adierazpide bitarrean oinarritzen da.

\section{BIBLIOGRAFIA}

[1] O'CONNOR J. J. eta ROBERTSON E. F. February 2018. MacTutor History of Mathematics archive, School of Mathematics and Statistics, University of St Andrews, Scotland. http://www-history.mcs.st-andrews.ac.uk/ Biographies/Stern.html

[2] STERN M. A. 1858. «Über eine zahlentheoretische Funktion,» Journal fur die reine und angewandte Mathematik, 55, 193-220.

[3] DIJKSTRA E.W. 1982. Selected Writings on Computing, Springer, p. 232.

[4] CALKIN N. eta WILF H. 2000. «Recounting the Rationals,» American Mathematical Monthly, volume 107, number 4, April 2000, 360-363. https:// www.math.upenn.edu/ wilf/website/recounting.pdf

[5] LEHMER D. H. 1929. «On Stern's Diatomic Series,» The American Mathematical Monthly, Vol.36, No. 2, Feb., 1929, pp. 59-67. http://www.jstor.org/ stable/2299356

[6] GIULI C. eta GIULI R. 1979. «A primer on Stern's diatomic sequence,» The Fibonacci Quarterly, 17, 103-108, 246-248 eta 318-320. https://www. fq.math.ca/Scanned/17-2/giuli.pdf

[7] URBIHA I. 2001. «Some properties of a function studied by De Rham, Carlitz and Dijkstra and its relation to the (Eisenstein-)Stern's diatomic sequence», Mathematical Communications 6, 181-198. https://core.ac.uk/ download/pdf/14375672.pdf

[8] NORTHSHIELD S. 2010. «Stern's Diatomic Sequence 0, 1, 1, 2, 1, 3, 2, 3, 1, 4,..» The American Mathematical Monthly 117 (7), 581-598. https:// digitalcommons.plattsburgh.edu/cgi/viewcontent.cgi?article $=1007 \&$ context $=$ mathematics_facpubs

[9] LANSING J. 2014, «Largest Values for the Stern Sequence,» Journal of Integer Sequences, 17, Article 14.7.5. https://cs.uwaterloo.ca/journals/JIS/ VOL17/Lansing/lansing2.pdf

[10] BANTCHEV B. B. 2012. «Fraction Space Revisited,» Mathematics and Education in Mathematics, Proc. 41th Spring Conf. of the Union of Bulgarian Mathematicians, April 2012, 209-218. http://arxiv.org/abs/1507.03952v1

[11] SLOANE N. J. A. 2018. The On-Line Encyclopedia of Integer Sequences (OEIS) (founded in 1964). https://oeis.org/A002487 
[12] R Core Team. 2018. R: A Language and Environment for Statistical Computing, Vienna, Austria. https://www.R-project.org

[13] M. J. CRAWLEY M. J. 2007. The R Book, John Wiley \& Sons, Ltd, England. 